\title{
Magnetic field emergence in quiet Sun granules
}

\author{
D. Orozco Suárez ${ }^{1}$, L. R. Bellot Rubio ${ }^{1}$, J. C. del Toro Iniesta ${ }^{1}$, and S. Tsuneta ${ }^{2}$ \\ 1 Instituto de Astrofísica de Andalucía (CSIC), Apdo. Correos 3004, 18080 Granada, Spain \\ e-mail: orozco@iaa.es \\ 2 National Astronomical Observatory of Japan, 2-21-1 Osawa, Mitaka, Tokyo 181-8588, Japan
}

Received 8 November 2007 / Accepted 15 December 2007

\section{ABSTRACT}

\begin{abstract}
Aims. We describe a new form of small-scale magnetic flux emergence in the quiet Sun. This process seems to take vertical magnetic fields from subsurface layers to the photosphere, where they appear above granular convection cells.

Methods. High-cadence time series of spectropolarimetric measurements obtained by Hinode in a quiet region near disk center are analyzed. We extract line parameters from the observed Stokes profiles and study their evolution with time.

Results. The circular polarization maps derived from the observed Fe I $630 \mathrm{~nm}$ lines show clear magnetic signals emerging at the center of granular cells. We do not find any evidence for linear polarization signals associated with these events. The magnetic flux patches grow with time, occupying a significant fraction of the granular area. The signals then fade until they disappear completely. The typical lifetime of these events is of the order of $20 \mathrm{~min}$. No significant changes in the chromosphere are seen to occur in response to the emergence, as revealed by co-spatial Ca II $\mathrm{H}$ filtergrams. The Stokes $I$ and $V$ profiles measured in the emerging flux concentrations show strong asymmetries and Doppler shifts.

Conclusions. The origin of these events is unclear at present, but we suggest that they may represent the emergence of vertical fields lines from the bottom of the photosphere, possibly dragged by the convective upflows of granules. Preliminary inversions of the Stokes spectra indicate that this scenario is compatible with the observations, although the emergence of vertical field lines is not free from conceptual problems.
\end{abstract}

Key words. Sun: magnetic fields - Sun: photosphere - instrumentation: high angular resolution

\section{Introduction}

Our knowledge of the quiet Sun magnetism has improved dramatically in recent years. We now have a relatively good understanding of the properties of the fields and other aspects such as the small-scale emergence and disappearance of magnetic flux concentrations. Analyses of spectropolarimetric data with moderate spatial resolution have significantly contributed to these topics. Lites et al. (1996), for example, discovered transient small-scale horizontal fields in quiet Sun areas. More recently, Martínez González et al. (2007) presented convincing evidence of low-lying loops connecting opposite-polarity flux concentrations in the solar internetwork. Using MDI longitudinal magnetograms at 1".2 resolution, Lamb et al. (2007) studied the emergence of apparently unipolar flux in the photosphere. Similar events have been detected in high-spatial resolution magnetograms obtained at the Swedish Vacuum Telescope (de Pontieu 2002).

Improvements in the spatial resolution of spectropolarimetric measurements allow these processes to be studied in greater detail. Using the spectropolarimeter aboard Hinode, Lites et al. (2007a,b) and Orozco Suárez et al. (2007a,b) found that internetwork fields tend to be horizontal with strengths below $0.5 \mathrm{kG}$. Also based on Hinode data, Centeno et al. (2007) and Ishikawa et al. (2008) confirmed the existence of very small-scale magnetic loops in quiet Sun areas and plage regions, respectively. The loops show horizontal fields above granules and footpoints of opposite polarity rooted in the adjacent intergranular lanes.
The frequency of appearance of these loops seems to be much higher than ever thought.

The characterization of emergence processes at the smallest scales is important for understanding the energy balance and origin of quiet Sun magnetic fields. In particular, they may hold the key to determine whether a local dynamo operates in the solar photosphere, as has been suggested on theoretical grounds (Cattaneo 1999). Also, a good knowledge of the ways magnetic fields emerge in the surface may help refine numerical simulations of magnetoconvection such as those performed by Vögler et al. (2005), Schaffenberger et al. (2006), Stein \& Nordlund (2006), and Abbett (2007). If some form of flux emergence is not observed in the simulations, additional ingredients might need to be implemented in current codes.

Here we analyze spectropolarimetric measurements of the quiet Sun taken with Hinode at a resolution of 0.'32. Several isolated, apparently unipolar, vertical field emergence events have been observed to occur in granules. We describe them and speculate on their origin in the next sections.

\section{Observations}

The data under analysis consists of two sequences of raster scans performed with the spectropolarimeter (SP; Lites et al. 2001) aboard Hinode (Kosugi et al. 2007). This instrument measures the Stokes profiles of the Fe I lines at $630.2 \mathrm{~nm}$ with a spectral sampling of $2.15 \mathrm{pm} \mathrm{pixel}^{-1}$ and a spatial sampling of 0 .' 16 . The sequences were taken on February 11 and March 10, 2007. In both cases, a narrow 4 " $\times 82^{\prime \prime}$ area at disk center was scanned 


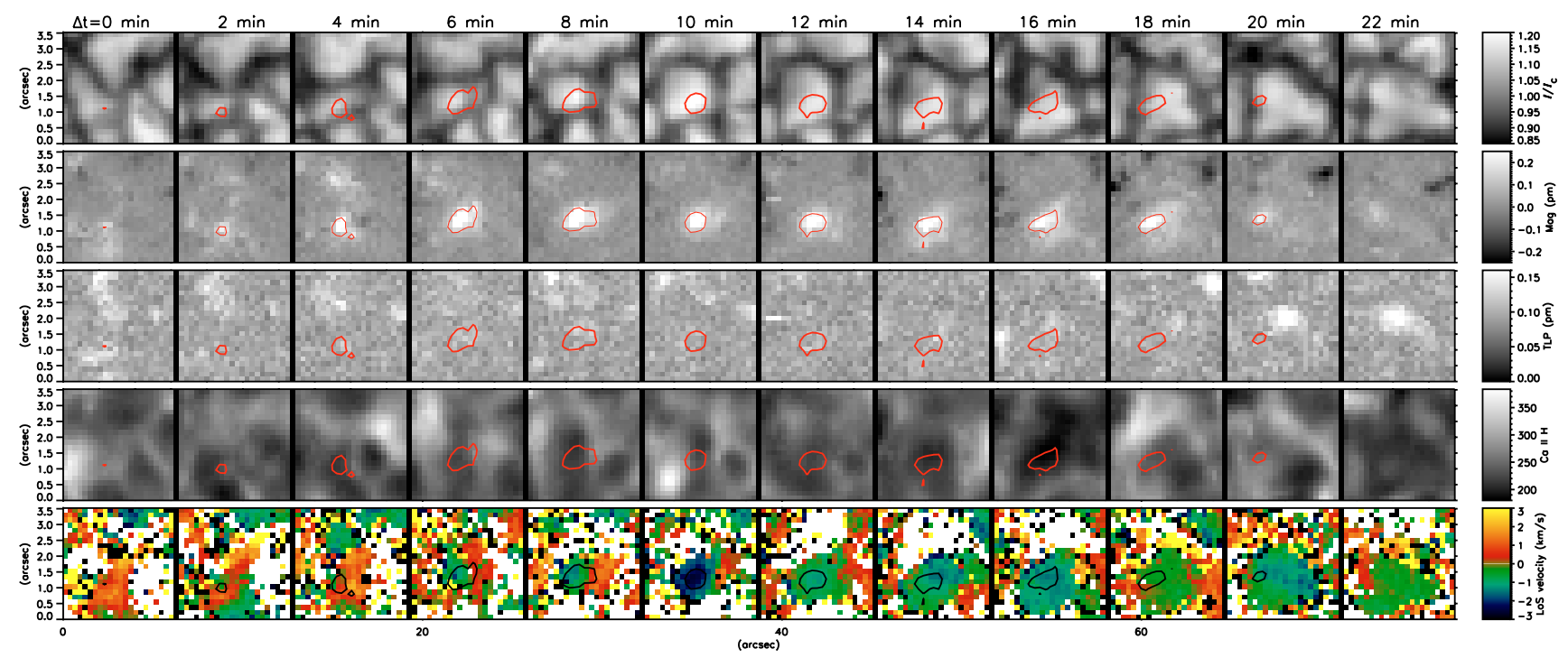

Fig. 1. Temporal evolution of a flux emergence process observed on February 11, 2007. The cadence is $123 \mathrm{~s}$ and the maps cover a FOV of 3.' $5 \times 3$ '. 5 . From top to bottom: normalized continuum intensity, circular polarization signal in the blue lobe of Fe I $630.25 \mathrm{~nm}$ (integrated over a wavelength range of $23 \mathrm{pm}$ centered on the peak position), total linear polarization signal $\left[\int\left(Q^{2}+U^{2}\right)^{1 / 2} \mathrm{~d} \lambda / I_{\mathrm{c}}\right]$, Ca II $\mathrm{H}$ line core intensity, and LOS velocity evaluated from the Stokes $V$ zero-crossing wavelengths. Negative velocities indicate blueshifts. Contours enclose areas with circular polarization signals larger than $0.15 \mathrm{pm}$. White areas represent pixels not included in the analysis because of their small signals. $\Delta t=0$ min corresponds to 11:07 UT.

repeatedly with a cadence of $123 \mathrm{~s}$. The integration time for each of the 25 slit positions was $4.8 \mathrm{~s}$, making it possible to achieve a noise level of $1.1 \times 10^{-3} I_{\mathrm{c}}$ in Stokes $V$ and $1.2 \times 10^{-3} I_{\mathrm{c}}$ in Stokes $Q$ and $U$. Filtergrams in the Ca II $\mathrm{H}$ line core were also acquired with the Hinode Broadband Filter Imager (BFI; Tarbell et al. 2007) to monitor the conditions of the chromosphere. The BFI pixel size was 0 ' $^{\prime} 054$ on February 11 and 0 ' $^{\prime} 108$ on March 10, and the cadences were 64 and $32 \mathrm{~s}$, respectively. Given that magnetic fields outside the photospheric network are not associated with enhanced $\mathrm{Ca}$ II $\mathrm{K}$ or $\mathrm{H}$ emission (Lites et al. 1999; Rezaei et al. 2007), the detection of transient $\mathrm{Ca}$ II $\mathrm{H}$ brightenings at or near the position of emerging flux would indicate that the emergence process is able to transfer a certain amount of energy to the (low) chromosphere.

The SP data have been corrected for dark current, flat-field, and instrumental cross-talk. The velocity scale for the Stokes spectra has been set by comparing the average quiet-Sun profile in each map with the FTS atlas, after subtraction of the gravitational redshift. The calibration algorithm applied to the filtergrams removed cosmic rays, hot pixels, and dark current. SP maps and BFI filtergrams have been aligned with sub-pixel accuracy following Shimizu et al. (2007).

\section{Results}

Figures 1 and 2 show the temporal evolution of two small-scale emergence events. Displayed are maps of continuum intensity at $630 \mathrm{~nm}$, circular and linear polarization signals, Ca II filtergrams, and LOS velocity maps. The total duration of the events is about 20 and $14 \mathrm{~min}$, respectively.

In the first event, the circular polarization maps show a unipolar flux concentration (white patch) barely visible at $\Delta t=$ 0 min. As time goes by, it grows both in size and strength. The maximum size and circular polarization signals are reached 8 min later. At that point, the flux concentration looks roundish and occupies an area of $\sim 4 \times 4$ pixels (some $200000 \mathrm{~km}^{2}$; red contours), which corresponds to one third of the granular cell surface. The granule is defined to be the region where the continuum intensity is at least 1.05 times brighter than the average quiet Sun. Then the signal starts to fade (16 min). At $\Delta t=24 \mathrm{~min}$ (not shown), the circular polarization signal has vanished completely. No clear opposite-polarity signals are detected in the observed area throughout the whole sequence. The continuum intensity maps demonstrate that the magnetic flux appears in an existing granule and persists there for 20 min while the granule evolves. Interestingly, the flux concentration does not seem to be disturbed by the granular flows: it remains co-spatial with the brightest part of the granule until $\Delta t=16 \mathrm{~min}$ and never gets advected to the adjacent intergranular lanes.

There is no detectable linear polarization signal associated with this event. Only the last two maps show traces of linear polarization when the circular polarization signal is almost absent. The observed linear polarization patch lies close to the flux concentration (less than $1^{\prime \prime}$ up and right), but we believe it is not related to its disappearance.

The emergence is characterized by blueshifted velocities from the initial stages. At $\Delta t=10 \mathrm{~min}$ we observe the stronger upflows of about $-2.5 \mathrm{~km} \mathrm{~s}^{-1}$. At that time, a chromospheric brightening is detected in the $\mathrm{Ca}$ II $\mathrm{H}$ filtergrams. It might be related to the emergence process, although it is not co-spatial with the flux concentration.

The second event differs slightly from the previous one. The magnetic feature appears at $\Delta t=2 \mathrm{~min}$ and persists for $12 \mathrm{~min}$ before it vanishes. The maximum spatial size of the magnetic patch is not larger than in the previous case. However, it occupies about half of the granule at $\Delta t=6 \mathrm{~min}$, and almost the whole granule 4 min later. The circular polarization signal is maximum at $\Delta t=14 \mathrm{~min}$. As before, there is no evidence for horizontal magnetic fields since no linear polarization is detected. The continuum intensity maps show that the granule starts to diminish in size at $\Delta t=10 \mathrm{~min}$, disappearing completely four minutes later. The flux concentration still persists, however. Thus, our observation suggests that the magnetic field somehow contributed to the granular disruption. Throughout the sequence, the flux concentration appears to be decoupled from the advection flow (e.g., it 


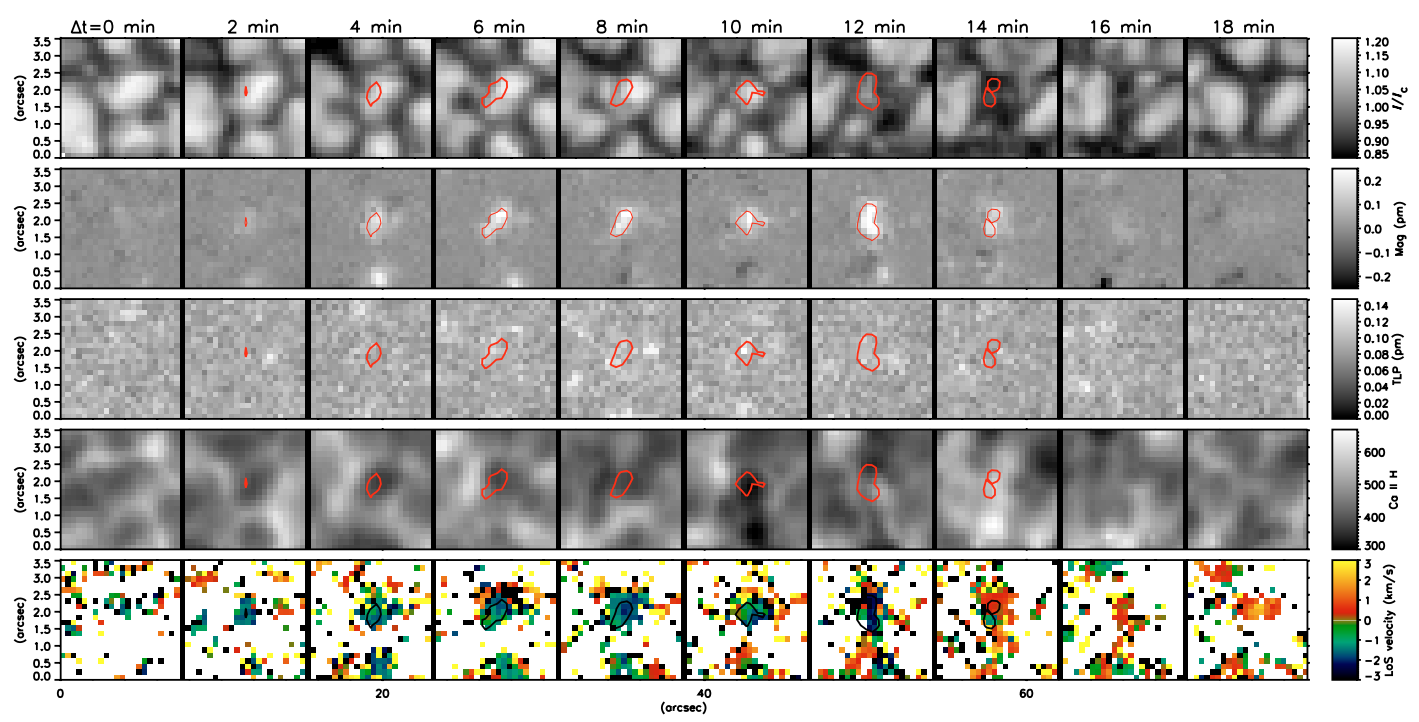

Fig. 2. Emergence event observed on March 10, 2007. The panels show the same quantities as those of Fig. $1 . \Delta t=0$ corresponds to 00:52 UT.

moves towards the center of the granule between $\Delta t=6 \mathrm{~min}$ and $\Delta t=10 \mathrm{~min})$.

Similarly to the first case, blueshifts are observed from the beginning of the process. The largest upflows of $-2.2 \mathrm{~km} \mathrm{~s}^{-1}$ occur at $\Delta t=12 \mathrm{~min}$. From $\Delta t=16 \mathrm{~min}$ on, weak downflows are detected instead, corresponding to the granule disappearance. The Ca II H line shows no significant brightenings associated with the process.

Figure 3 displays Stokes $I$ and $V$ profiles observed during the first event. They correspond to the center of the flux concentration at $\Delta t=8,10$ and $12 \mathrm{~min}$ (solid, dotted, and dashed lines, respectively). We do not include Stokes $Q$ and $U$ spectra because their signals are below the noise.

Both Stokes $I$ and $V$ exhibit strong asymmetries at $\Delta t=$ $8 \mathrm{~min}$. The blue wing of Stokes $I$ is significantly blueshifted while the line core remains almost at rest, suggesting strong upflows in deep atmospheric layers and smaller velocities higher up. The signature of large gradients of atmospheric parameters is even more conspicuous in Stokes $V$ : since the pioneering work by Illing et al. (1975) and Auer \& Heasley (1978) we know that the circular polarization profiles are symmetric unless a velocity gradient is present along the LOS. The asymmetry of Stokes $V$ is extreme in this case, with the red lobe being almost absent. Such a degree of asymmetry can only be produced by large velocity and magnetic field gradients. At $\Delta t=10 \mathrm{~min}$, the whole line is affected by a strong blueshift, but the velocity gradient seems to have decreased significantly since the profiles look more symmetric. At $\Delta t=12 \mathrm{~min}$, the gradients are still small and the global velocity shift is reduced. Altogether, this qualitative interpretation of the Stokes $I$ and $V$ profiles suggests that we are witnessing the rise of magnetic fields through the granule, from the bottom of the photosphere to higher layers. Apparently, the field is vertical because we do not detect linear polarization signals.

\section{Preliminary inversions}

We have inverted the profiles displayed in Fig. 3 to estimate the field strength and flow velocity in the emerging magnetic flux concentration. The inversions were carried out with SIRJUMP, a modified version of the SIR code (Ruiz Cobo \& del Toro Iniesta 1992). Unlike its parent, SIRJUMP is able to deal with discontinuous stratifications along the LOS. The discontinuity is modeled
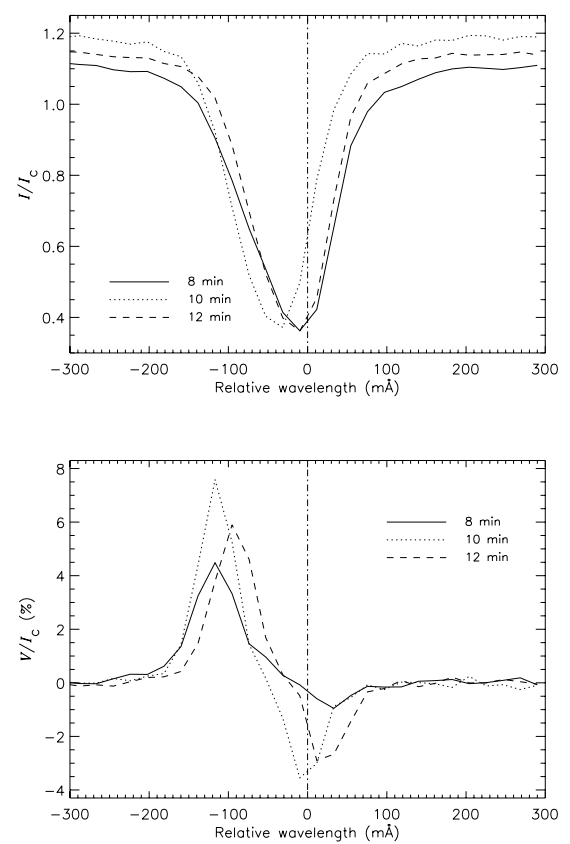

Fig. 3. Stokes $I$ (top) and $V$ (bottom) profiles of Fe I $630.25 \mathrm{~nm}$ observed at the center of the flux concentration studied in Fig. 1. The solid, dotted and dashed lines correspond to $\Delta t=8,10$, and $12 \mathrm{~min}$, respectively. The vertical lines mark the zero point of the velocity scale.

as a step function. Tests with the code show that it successfully distinguishes cases where a discontinuity is needed from those that do not require it. We run SIRJUMP with one-component model atmospheres and unity filling factors, allowing for a local stray light contamination as described by Orozco Suárez et al. (2007a,b).

The results of these preliminary inversions indicate that the only way to reproduce the Stokes $I$ and $V$ profiles observed at $\Delta t=8 \mathrm{~min}$ is by means of a discontinuous stratification of magnetic field strength. A weak vertical field of about $250 \mathrm{G}$ is present in the lower photosphere, together with strong upflows of some $-2.5 \mathrm{~km} \mathrm{~s}^{-1}$. The field strength is negligible above $\log \tau_{5} \sim-1$. The discontinuity moves to higher layers 
at $\Delta t=10 \mathrm{~min}$ and eventually leaves the line-forming region at $\Delta t=12 \mathrm{~min}$. The scenario coming out from the inversions is therefore compatible with the qualitative suggestions made from the shapes of the profiles: in the first stages of the emergence we could be seeing the ascent of magnetic fields that do not fill the line-forming region completely. Once the fields reach the upper photosphere, the gradients of atmospheric parameters decrease significantly and, as a consequence, the Stokes profiles become less asymmetric.

\section{Discussion and conclusions}

We have described here two cases of the emergence of apparently unipolar, vertical fields in granular cells. A total of six such events occurred during the $10 \mathrm{~h}$ covered by the Hinode observations. Their lifetimes are on the order of 15-20 min.

To the best of our knowledge, this form of small-scale magnetic flux emergence has not been described in the literature. It differs significantly from the emergence processes in granular convection studied by Centeno et al. (2007) and Ishikawa et al. (2008), since we do not detect linear polarization signals or opposite-polarity footpoints surrounding them. The geometry of the fields we observe is not that of small magnetic loops. Lamb et al. (2007) have described examples of the emergence of unipolar flux, but at a poorer resolution of 1 '.2. No association of the flux with granules or intergranules was made in their paper. They suggested that the origin of the unipolar flux appearance is coalescence of pre-existing field lines with the same polarity, which were below the detection limit imposed by the intrinsic noise and the spatial resolution of their observations. While it is not possible for us to rule out the scenario of field-line coalescence, we do not find evidence for diffuse magnetic fields in the emergence sites prior to the events, at the much higher spatial resolution and sensitivity of Hinode.

Current magnetoconvection simulations do not seem to explain our observations either. The simulations of Vögler et al. (2005) do show magnetic fields in granules, but they are much weaker than those reported here, and do not undergo emergence processes. Those granular fields may be the result of recycling of flux initially placed in intergranular lanes, or an effect of enhanced magnetic diffusivities. Cheung et al. (2007), on the other hand, studied the rise of magnetic flux tubes from the convection zone to the photosphere. Depending on the magnetic flux stored in the tubes, the arrival of magnetic fields at the solar surface has very different observable consequences. For the stronger tubes, a darkening and distortion of the granular convection is expected (and actually observed), while weaker tubes do not modify the brightness of surface granules. In both cases, magnetic fields tend to emerge at the center of granular cells, showing large inclinations to the vertical. The fields are then advected by the horizontal flow towards the intergranular lanes, where they become more vertical and form opposite-polarity patches. Our events do not share these properties.

A hypothetical scenario for the emergence of unipolar vertical magnetic fields would be that granular upflows drag horizontal field lines initially placed in the upper convection zone, carrying them to the photosphere where they would emerge in the granules. However, it is not clear how the horizontal fields could turn into vertical fields. Also, at some point one should observe opposite polarities where the field lines return to the solar surface, but we do not detect them, perhaps as a consequence of still insufficient sensitivity or because they occur outside of the FOV. What is clear is that the scenario of vertical fields emerging in granules faces important conceptual problems.

A radically different interpretation is that the events we have observed do not involve the emergence of new flux, but the "excitation" of already existing, mixed, quasy-isotropic fields (López Ariste et al. 2008). These fields would be largely decoupled from convective motions and hence not affected by them. They do not need to be purely vertical: if the degree of mixing is sufficiently high, inclined fields may also produce negligible linear polarization signals. This scenario should be investigated more thoroughly, both to demonstrate the existence of such tangled fields and to assess whether they are indeed compatible with the observations presented here.

In summary, at this stage we cannot offer a clear explanation of the events observed with Hinode, but we hope that the report of such a process will stimulate further observational and theoretical work. With this in mind, we plan to perform detailed inversions of the observed profiles in an attempt to derive a consistent picture of the physics behind these processes. Since sensitivity may be an issue, it would also be convenient to carry out additional measurements with the Hinode SP, pushing the integration time to a limit.

Acknowledgements. Hinode is a Japanese mission developed and launched by ISAS/JAXA, with NAOJ as domestic partner and NASA and STFC (UK) as international partners. It is operated by these agencies in co-operation with ESA and NSC (Norway). This work has been partially funded by the Spanish Ministerio de Educación y Ciencia through project ESP2006-13030-C06-02 (including European FEDER funds).

\section{References}

Abbett, W. P. 2007, ApJ, 665, 1469

Auer, L. H., \& Heasley, J. N. 1978, A\&A, 64, 67

Cattaneo, F. 1999, ApJ, 515, L39

Centeno, R., Socas-Navarro, H., Lites, B., et al. 2007, ApJ, 666, L137

Cheung, M. C. M., Schüssler, M., \& Moreno-Insertis, F. 2007, A\&A, 467, 703 de Pontieu, B. 2002, ApJ, 569, 474

Illing, R. M. E., Landman, D. A., \& Mickey, D. L. 1975, A\&A, 41, 183

Ishikawa, R., Tsuneta, S., Ichimoto, K., et al. 2008, A\&A, 481, L25

Kosugi, T., Matsuzaki, K., Sakao, T., et al. 2007, Sol. Phys., 243, 3

Lamb, D. A., DeForest, H. J., Hagenaar, H. J., Parnell, C. E., \& Welsch, B. T. 2007, ApJ, in press [preprint doi: 10.1086/'524372']

Lites, B. W., ocas Navarro, H., Kubo, M., et al. 2007a, PASJ, 59, L571

Lites, B. W., Socas Navarro, H., Kubo, M., et al. 2007b, ApJ, 672, L1237

Lites, B. W., Leka, K. D., Skumanich, A., Martínez Pillet, V., \& Shimizu, T. 1996, ApJ, 460, 1019

Lites, B. W., Rutten, R. J., \& Berger, T. E. 1999, ApJ, 517, 1013

Lites, B. W., Elmore, D. F., \& Streander, K. V. 2001, ASP Conf. Ser., 236, 33

López Ariste, A., et al. 2008, in Proceedings of the Solar Polarization Workshop 5 , ed. S. Berdyugina et al., ASP Conf. Ser., in preparation

Martínez González, M. J., Collados, M., Ruiz Cobo, B., \& Solanki, S. K. 2007, A\&A, 469, L39

Orozco Suárez, D., Bellot Rubio, L. R., del Toro Iniesta, J. C., et al. 2007a, ApJ, 670, L61

Orozco Suárez, D., et al. 2007b, PASJ, 59, in press

[arXiv: astro-ph/0709.2033]

Rezaei, R., Schlichenmaier, R., Beck, C. A. R., Bruls, J. H. M. J., \& Schmidt, W. 2007, A\&A, 466, 1131

Ruiz Cobo, B., \& del Toro Iniesta, J. C. 1992, ApJ, 398, 375

Schaffenberger, W., Wedemeyer-Böhm, S., Steiner, O., \& Freytag, B. 2006, in Solar MHD: Theory and Observations - a High Spatial Resolution Perspective, ed. J. Leibacher, H. Uitenbroek, \& R. F. Stein, ASP Conf. Ser., 354,345

Shimizu, T., et al. 2007b, PASJ, 59, in press

Stein, R. F., \& Nordlund, Å. 2006, ApJ, 642, 1246

Tarbell, T. D., et al. 2007, Sol. Phys., in preparation

Vögler, A., Shelyag, S., Schüssler, M., et al. 2005, A\&A, 429, 335 